Journal of Soft Computing Paradigm (JSCP) (2020)

Vol.02/ No. 01

Pages: $13-23$

http://irojournals.com/jscp/

DOI: https://doi.org/10.36548/jscp.2020.1.002

\title{
Intelligent and Adaptive Multi-Objective Optimization in WANET Using Bio Inspired Algorithms
}

\author{
Dr. Subarna Shakya \\ Professor, Department of Electronics and Computer Engineering, \\ Central Campus, Institute of Engineering, Pulchowk, \\ Tribhuvan University, \\ Pulchowk, Lalitpur Nepal. \\ Email: drss@ioe.edu.np
}

\begin{abstract}
A WANET is a specific type of Adhoc network with a community of specialized, self-organizing mobile devices. Because of the complex existence of mobile devices and wireless networking, the adhoc network communicating in wireless manner faces many problems related to the topological organizations, battery capacity limitations that results in energy restrictions, transmission of information, etc. The significant attributes of an adhoc network communicating in wireless manner are the quality of service and the percentage of energy used. Nowadays as the bio-inspired computing plays a major role in the research field. Specifically bio-inspired algorithms motivated by nature's actions are used immensely to accomplish optimization in issues faced in communication. Massive works have been reported in the recent years to enhance the quality of service utilizing the Bio-inspired computing due to its intelligent and adaptive nature. The paper proposes the intelligent and an adaptive routing employing the bio inspired genetic bee colony algorithm and the ant colony optimization to have optimized energy utilization maximizing the longevity of the network. The proposed method is evaluated suing the network simulator-2 on the basis of the energy utilization, longevity and the service quality (Throughput) of the network, the results observed for the method put forth were better compared to the existing methods.
\end{abstract}

Keyword: Adhoc Network, Wireless Adhoc Network (WANET), Bio Inspired Computing, Genetic Bee Colony Algorithm, Adaptive and Intelligent Routing

\section{Introduction}

ISSN: 2582-2640 (online) 
Journal of Soft Computing Paradigm (JSCP) (2020)

Vol.02/ No. 01

Pages: $13-23$

http://irojournals.com/jscp/

DOI: https://doi.org/10.36548/jscp.2020.1.002

The adhoc networks communicating in wireless manner are enriched with multitude of capabilities such as self-organizing, self - healing or reconfiguring on cases of sudden failures in the single devices in the network. These devices are capable of framing network with devices that hold varying computational capacities, memory and battery availability, so the communication could be extended with the devices that are both heterogeneous and homogenous. The recent developments in the mobile devices have been the main reason for the development of these types of network framed in adhoc manner. These adhoc type of networks as the name suggests were structured without a central body in the wireless medium just for a specific reasons.

The places where proper management is essential due to the natural disasters such as catastrophe, manmade crisis that includes accidents, terrorist activities etc., and in the situations where a assistance for a vehicle is essential, all these circumstances requires immediate communication mode to receive the necessary response, and the adhoc network that could be made possible with the mobile device that are available around seems more promising in such situations as it allows a two a communication utilizing the volunteering neighboring mobile devices. These networks are capable of being formed even in places that lacks a properly organized and network foundations.

Since these system are structured on time of need, they are not centralized and does not possess a proper topology, and often changes it topology as they are formed using the devices that are mobile, more over the incapability of the each devices in transmitting to a very long distance make them to convey the information's taking multiple strides utilizing the intermediate nodes as the conveyors. These network functions are backed by the battery power of the devices that are added to the network. So the longevity of the network depends on the available power in each devices in the network that are added to the network. In adhoc networks communicating through wireless medium, the portable nodes are automatically sorted out and configured to automatically expand and safeguard the entire network framework; in this way, the guiding philosophies are central. Owing to dynamic growth and restricted funds, maintaining a vibrant system capable of expertly seeking a multipath with minimal overhead traffic is incredibly big in the WANETS. In spite of the vast advancements in the battery in order to extend the life of the devices the energy still a restricted access due to the widespread utilization in the of the wireless adhoc networks.

So to communicate using the adhoc network from the source to destination it becomes necessary to identify the possible pathway available between the source and the destination through the mobile devices that are included to the network and it's essential to identify a most reliable path with devices of maximum battery

ISSN: 2582-2640 (online) 
Journal of Soft Computing Paradigm (JSCP) (2020)

Vol.02/ No. 01

Pages: $13-23$

http://irojournals.com/jscp/

DOI: https://doi.org/10.36548/jscp.2020.1.002

power, least overhead for communication, and minimum distance to the destination in order to have an optimized energy that extends the longevity of the network as well as the path identified.

So to identify the perfect device is one of the most essential need of the adhoc network as major portion of the energy are consumed in identify the device that are suitable, so the proposed method puts forth the genetic bee colony algorithm to identify the suitable devices and employs the ant colony algorithm to sort out the suitable shortest path. The left behind paper is prepared with the related works in the part 2, the proposed GBC based suitable device identification and ACO based path identification in the part 3 and 4 the results evaluation in part 5 and the conclusion in part 6.

\section{Related Works}

Bajaber et al [1] proposes the "Energy efficient clustering protocol to enhance lifetime of wireless sensor network." Raj, Jennifer S.et al [2] puts forth the novel method to have a better quality of service in the wireless sensor engaged in the internet of things by proposing an energy efficient routing. De Rango, et al [3] elaborates the formation of routing using the ant's behavior for saving the rate of energy utilization and have balanced load in the adhoc networks that communicate in the wireless medium. Smys, S.et al [4] I his paper has proposed a "secure routing protocol with the energy optimization for the wireless sensor networks" to enhance the reliability in the path by engaging the nodes that are highly trusted.

Sathesh, A. et al [5] in the recent years proposed the "Optimized Multi-Objective Routing for Wireless Communication with Load Balancing." To minimize the energy and develop a stable path between the source and destination. Khabiri et al [6] has utilized the cuckoo search algorithm to bring down the energy utilized in the cluster formation and path tailing in the wireless sensor networks. Bhalaji, $\mathrm{N}$ et al [7] proposes a "Performance Evaluation of Flying Wireless Network With Vanet Routing Protocol." Qu, et al [8] does the routing to optimize the energy, utilization in the wireless sensor networks by employing the ant colony optimization.

Pandian, M. Durai. et al [9] proposed an ""Enhanced Network Selection and Handover Schema for Heterogeneous Wireless Networks." Smys, S., and Jennifer S. Raj et al [10] put forth the "A Stochastic Mobile Data Traffic Model for Vehicular Ad Hoc Networks." Sivaganesan, D et al [11] proffered a "Efficient Routing Protocol With Collision Avoidance In Vehicular Networks." Haoxiang et al [12] put forth the novel concept of. "Multi-Objective Optimization Algorithm for Power Management in Cognitive

ISSN: 2582-2640 (online)

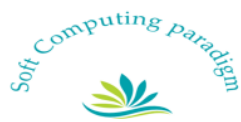


Journal of Soft Computing Paradigm (JSCP) (2020)

Vol.02/ No. 01

Pages: $13-23$

http://irojournals.com/jscp/

DOI: https://doi.org/10.36548/jscp.2020.1.002

Radio Networks." Smys, S., and Jennifer S. Raj. et al [12] an authenticated routing with the energy management for the wireless adhoc networks,

Duraipandian, M. proposed a machine learning based routing for mobile adhoc networks and evaluated its performance in terms of energy and the network longevity, Wang, et al [15] in his paper presented the method of optimized routing by employing the soft computing strategies such as the fuzzy petri-net. Alshamlan, et al [16] in his paper proposes the Genetic Bee Colony (GBC) algorithm: A new gene selection method for microarray cancer classification."

The proposed method in the paper utilizes the GBC algorithm to select the optimal devices that are long lasting with minimum mobility, maximum energy availability and minimum distance to the destination and engages the ACO to sort out the routes with the shortest distance to have an energy efficient communication that enhances the longevity and the service quality of the network.

\section{Proposed Work}

The paper aims in devising an energy efficient routing that improvises the longevity of the network and its service quality. Minimizing the energy in the wireless adhoc network is multi facet problem as the energy utilization depends on the mobility of the devices $\left(D_{M}\right)$, the distance of the devices to its neighboring devices $\left(D_{D i-N D}\right)$, distances to the destination $\left(D_{D i-D e}\right)$, the distance to the source $\left(D_{D i-S}\right)$, and the energy availability of the device $\left(D_{E}\right)$, apart from this the energy utilization also depends on the distance of the path to the destination, its band width utilization and the communication overhead, etc., So minimizing the energy utilization the wireless adhoc networks becomes an multi-objective problem. The proffered method in the paper optimizes he multi-objective problem using the GBC to select the optimal devices and uses the ACO to select the shortest path.

\subsection{Optimal Device Selection for Wireless Adhoc Using GPC}

The Genetic Bee Colony [16] is a combination of the genetic and the artificial bee colony algorithm developed with the aim of selecting highly optimal device so as to minimize the overall energy utilization in the wireless adhoc networks, the figure. 1 below is the general flowchart of the genetic bee algorithm.

ISSN: 2582-2640 (online)

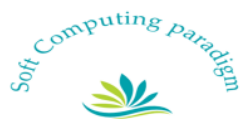


Journal of Soft Computing Paradigm (JSCP) (2020)

Vol.02/ No. 01

Pages: $13-23$

http://irojournals.com/jscp/

DOI: https://doi.org/10.36548/jscp.2020.1.002

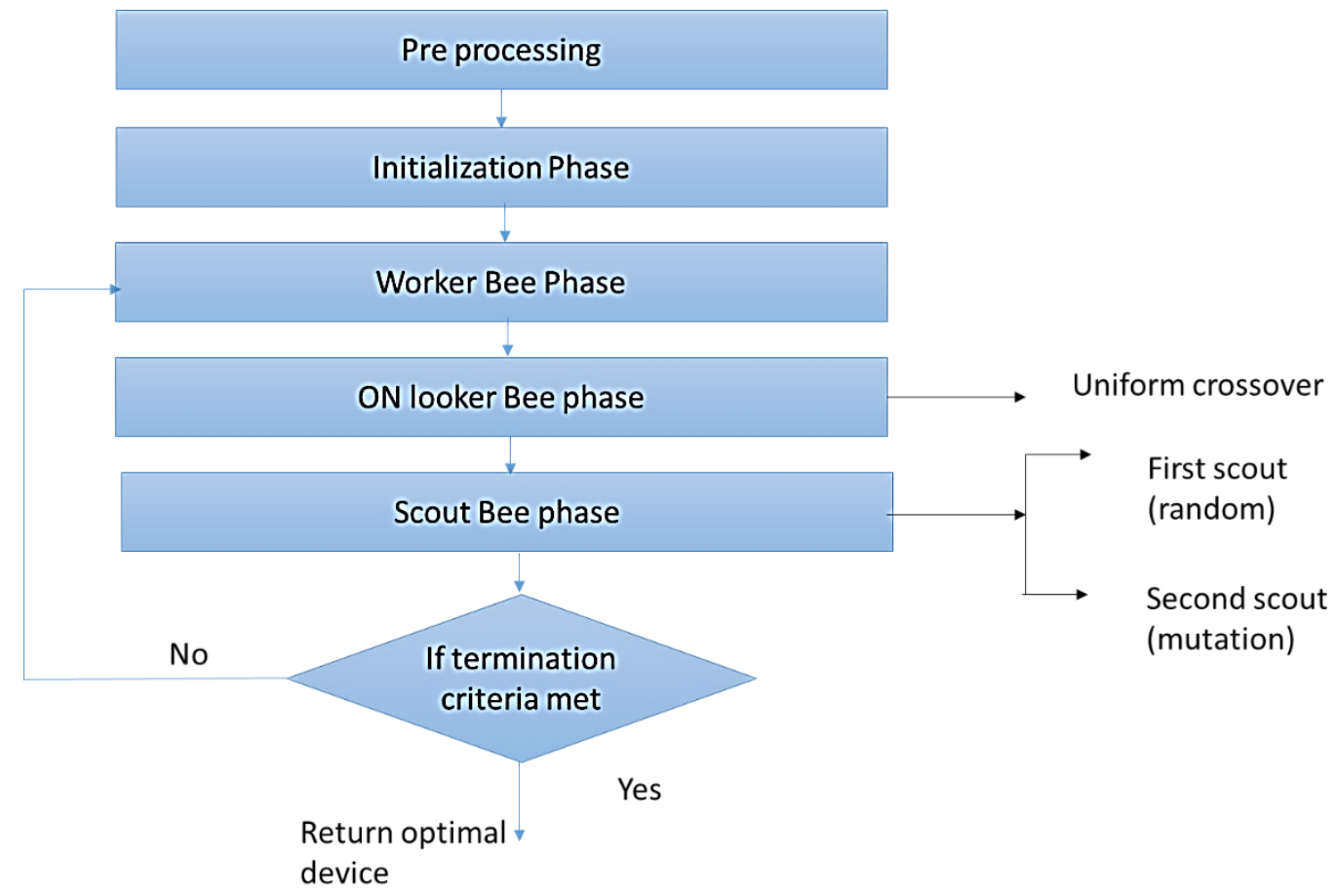

Figure.1 Flow Chart of GBC [16]

The pre-convergence problem of the genetic algorithm as well as the poor solution exploitation and the extended computation time of the artificial bee colony algorithm are overcome by combining the GA and the $\mathrm{ABC}$, the information sharing in the $\mathrm{ABC}$, among the onlooker bees and the worker bees and the procedures to swap the worn-out solution in the scout bee phase are improved by engaging the genetic operators this is termed as the GBC, the GBC identifies the optimal devices by selecting the devices with maximum $\left\{D_{E}\right\} \cup$ minimum $\left\{D_{D i-N D} \cup D_{M}\right\}$ for forming the network, every node transmits its deviceID along with the particulars that are aforementioned for the network to find the devices that are optimal. Once the optimal device are found the network is formed with the suitable nodes, updating the information's of the devices in the network to the database that holds the particulars of the devices.

ISSN: 2582-2640 (online) 
Journal of Soft Computing Paradigm (JSCP) (2020)

Vol.02/ No. 01

Pages: $13-23$

http://irojournals.com/jscp/

DOI: https://doi.org/10.36548/jscp.2020.1.002

\subsection{Optimal Path Selection Applying ACO}

Once the optimal devices are selected and the network is formed, the source that request for the transmission of the information's to the destination beyond the reach tries find out a suitable path that has minimum bandwidth utilization, communication overhead and distance to the destination, in order to minimize the energy utilization. The source device identifies the possible path between the source and destination and updates to the database holding the particulars of the path and enumerates the shortest path applying he ACO that uses the foraging behavior of the ants to select a shortest path by selecting path enriched with device with the maximum $\left(\left\{D_{E}\right\} \cup\left\{D_{D i-S}\right\}\right)$ minimum $\left(\left\{D_{D i-N D}\right\} \cup\left\{D_{D i-D e}\right\}\right)$ and with minimum $\left(\left\{B_{W}\right\} \cup\left\{C_{O H}\right\}\right)$ and discards the remaining. So the next transmission taking place checks for the available possible paths and if not suitable begins to collect the information of the possible path.

\section{Proposed GBC -ACO based Optimized Routing for WANET}

The proposed GBC-ACO based route selection for the wireless adhoc network is explained in the step wise description given below.

As these type of networks are formed only when it is necessary the source device whenever it has the need to transmit the information forms the network by generating Requesting message, the willing mobile devices reply back with the identification and the particular of it to the source devices.

1. The source that has to start it transmission sends request to get details about its neighboring devices.

2. The willing devices replies back with the $\left\{\left(D_{I D 1}\right.\right.$, Device particulars $)$, ( $D_{I D 2}$, Device particulars $) \ldots\left(D_{I D n}\right.$, Device Particulars $\left.)\right\}$

3. The source collects the $\left\{D_{M}, D_{D i-N D}, D_{D i-D e}, D_{D i-S}, D_{E}\right\}$ for every devices

4. Enumerates the suitability of the devices applying the GBC for all devices

5. Finds out devices with the maximum $\left\{D_{E}\right\} \cup \operatorname{minimum}\left\{D_{D i-N D} \cup D_{M}\right\}$

6. Updates to the database

7. The source identifies all the possible paths existing between the source to destination

8. Determines the shortest path by finding out paths with maximum $\left(\left\{D_{E}\right\} \cup\left\{D_{D i-S}\right\}\right)$ minimum $\left(\left\{D_{D i-N D}\right\} \cup\left\{D_{D i-D e}\right\}\right)$ and with minimum $\left(\left\{B_{W}\right\} \cup\left\{C_{O H}\right\}\right)$ Applying ACO

9. Updates the shortest path to the table

ISSN: 2582-2640 (online)

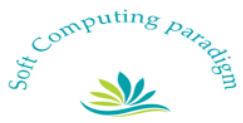


Journal of Soft Computing Paradigm (JSCP) (2020)

Vol.02/ No. 01

Pages: $13-23$

http://irojournals.com/jscp/

DOI: https://doi.org/10.36548/jscp.2020.1.002

10. Conveys information to the destination, on initializing the new transmission the source checks for the previous path if available uses it else goes to step 1.

The information's of the devices in the data base are periodically updated to know about the devices that has left the network and the new devices available in the network.

\section{Result Evaluation}

The proposed method is simulated in network simulator-2 for varying number of mobile devices ranging from $100-500$, within a simulation range of the 2500meters, and the initial energy of 100 joules per device with the packet size ranging between 1000 to 2000 bytes. The results for the proposed method and the existing methods on the basis of energy utilization, longevity and the service quality were evaluated and compared.

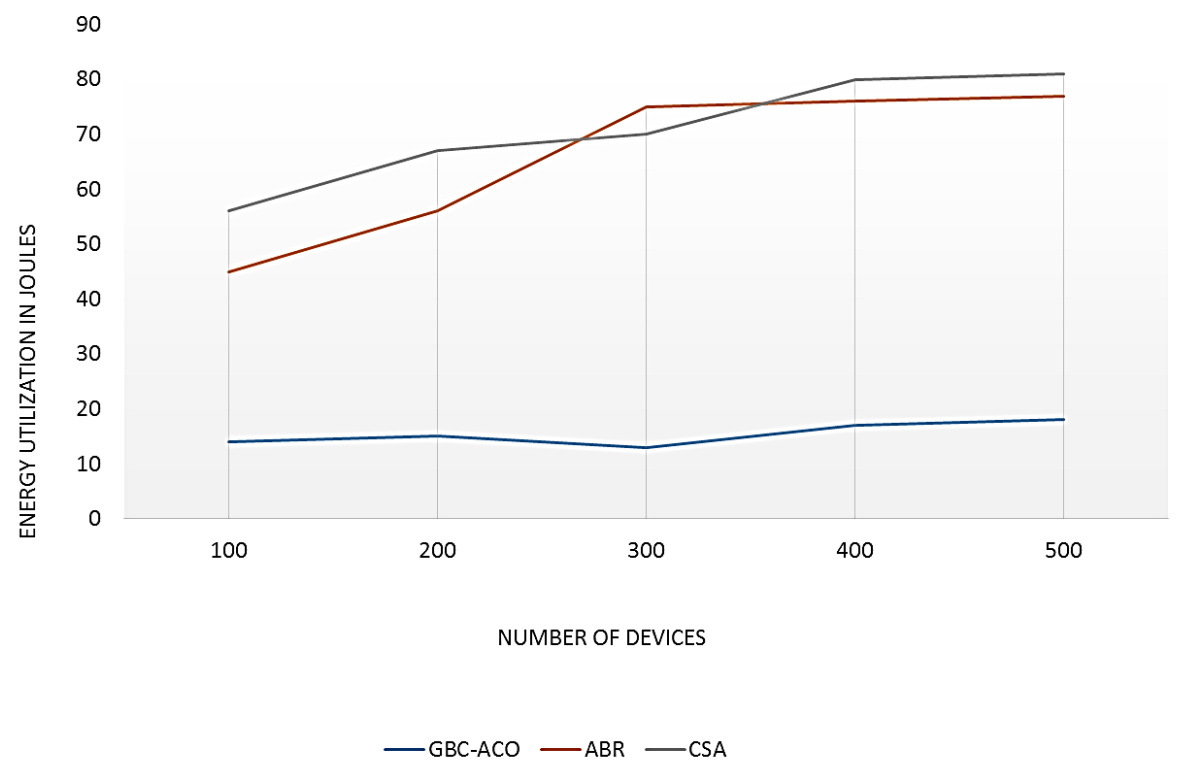

Figure. 2 Energy utilization

ISSN: 2582-2640 (online) 
Journal of Soft Computing Paradigm (JSCP) (2020)

Vol.02/ No. 01

Pages: 13-23

http://irojournals.com/jscp/

DOI: https://doi.org/10.36548/jscp.2020.1.002

The results observed on the energy utilization is depicted in the figure. 2 above the results show that the proposed method shows a considerable reduction in the energy utilization, compared to the existing cuckoo search algorithm [6] and the ant based routing [3]

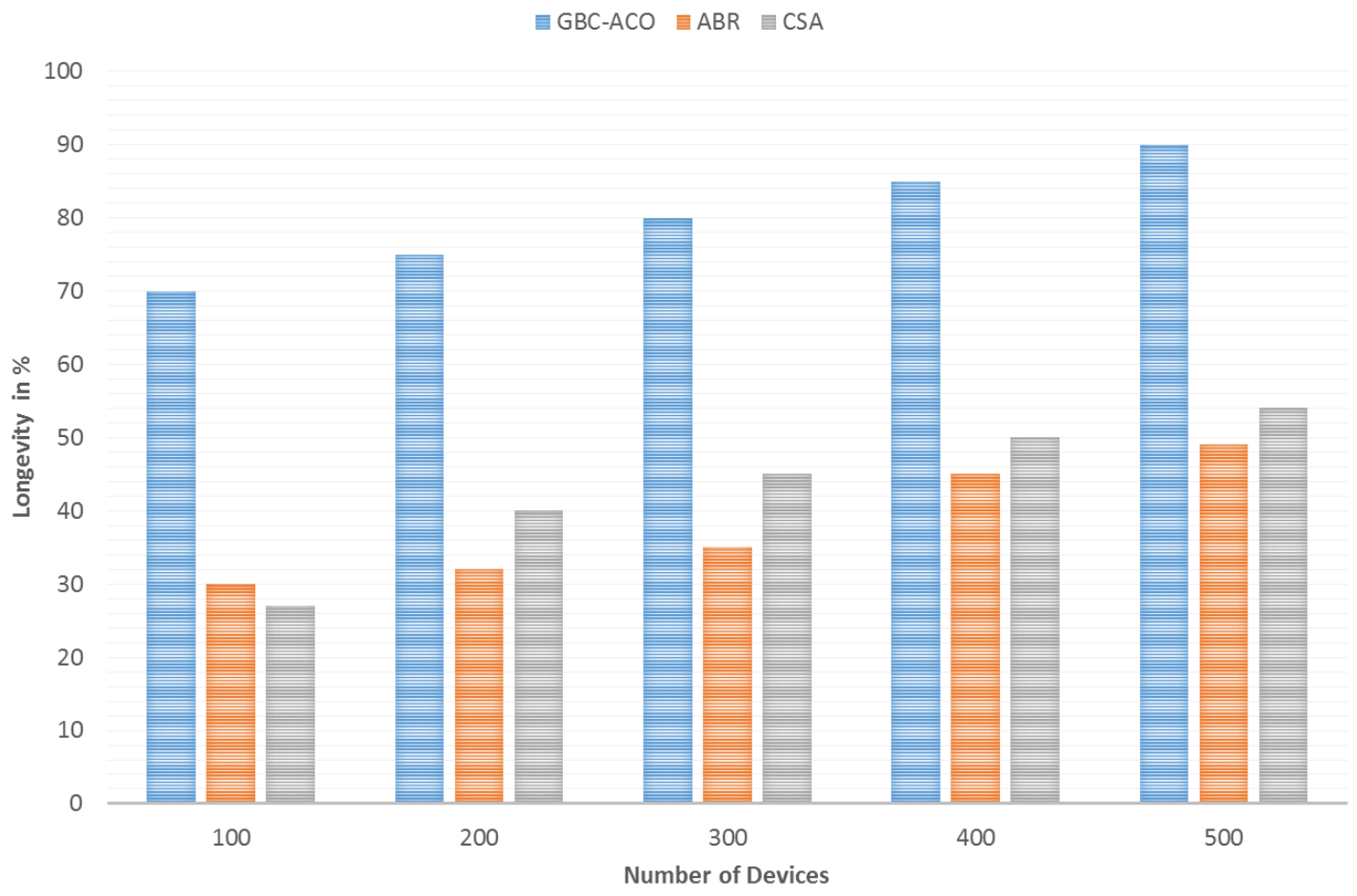

Figure. 3 longevity of Network

The results observed on the longevity of network is depicted in the figure. 3 above the results show that the proposed method shows a betterment in the longevity of Network, compared to the existing cuckoo search algorithm and the ant based routing.

ISSN: 2582-2640 (online)

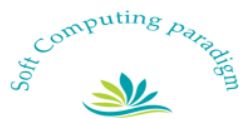


Journal of Soft Computing Paradigm (JSCP) (2020)

Vol.02/ No. 01

Pages: $13-23$

http://irojournals.com/jscp/

DOI: https://doi.org/10.36548/jscp.2020.1.002

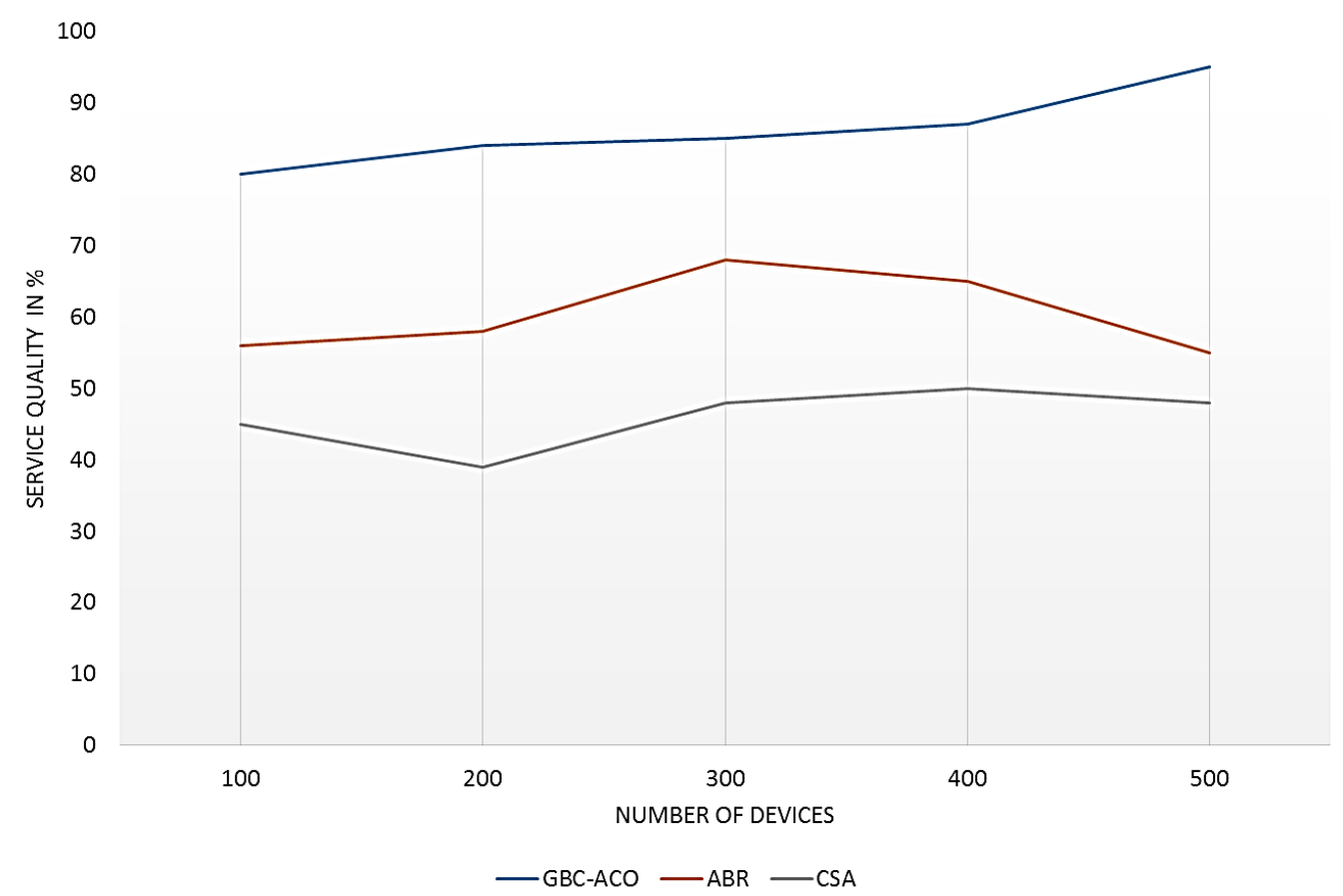

Figure. 4 Service Quality

The quality of the service for the network is decided based on the throughput of the network, the figure .4 shows the percentage of the service quality attained in the network for varying number of devices, the results shows that the percentage of service quality of the proposed method is much better compared to the existing cuckoo search algorithm and the ant based routing.

\section{Conclusion}

The bio inspired methods such as the GPC and the ACO is employed in the proposed method to select the more optimal devices and the path respectively for conveying the information from source to the destination on an adhoc network that communicates without wires. The proposed method was simulated using the network simulator-2 along with the existing methodologies and the results proved that the proposed method showed better results compared to the exiting cuckoo

ISSN: 2582-2640 (online)

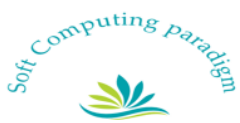


Journal of Soft Computing Paradigm (JSCP) (2020)

Vol.02/ No. 01

Pages: $13-23$

http://irojournals.com/jscp/

DOI: https://doi.org/10.36548/jscp.2020.1.002

search algorithm and the ant based algorithm. The future work of the paper is to enumerate the trust of the nodes make the communication more secured.

\section{References}

[1] Bajaber, Fuad, and Irfan Awan. "Energy efficient clustering protocol to enhance lifetime of wireless sensor network." Journal of Ambient Intelligence and Humanized Computing 1, no. 4 (2010): 239-248.

[2] Raj, Jennifer S. "QoS optimization of energy efficient routing in IoT wireless sensor networks." Journal of ISMAC 1, no. 01 (2019): 12-23.

[3] De Rango, Floriano, and Mauro Tropea. "Energy saving and load balancing in wireless ad hoc networks through ant-based routing." In 2009 International Symposium on Performance Evaluation of Computer \& Telecommunication Systems, vol. 41, pp. 117-124. IEEE, 2009.

[4] Smys, S. "Energy-Aware Security Routing Protocol For Wsn In Big-Data Applications." Journal of ISMAC 1, no. 01 (2019): 38-55.

[5] Sathesh, A. (2019). Optimized Multi-Objective Routing For Wireless Communication With Load Balancing. Journal of trends in Computer Science and Smart technology (TCSST), 1(02), 106-120.

[6] Khabiri, Melika, and Ali Ghaffari. "Energy-aware clustering-based routing in wireless sensor networks using cuckoo optimization algorithm." Wireless Personal Communications 98, no. 3 (2018): 2473-2495.

[7] Bhalaji, N. "Performance Evaluation of Flying Wireless Network With Vanet Routing Protocol." Journal of ISMAC 1, no. 01 (2019): 56-71.

[8] Qu, Wei, and Xiaowei Wang. "An Energy-Saving Routing Strategy Based on Ant Colony Optimization in Wireless Sensor Networks." In International Conference on Swarm Intelligence, pp. 277-284. Springer, Cham, 2017.

[9] Pandian, M. Durai. "Enhanced Network Selection and Handover Schema for Heterogeneous Wireless Networks." Journal of ISMAC 1, no. 03 (2019): 160-171.

[10] Malar, A. Christy Jeba, M. Kowsigan, N. Krishnamoorthy, S. Karthick, E. Prabhu, and K. Venkatachalam. "Multi constraints applied energy efficient routing technique based on ant colony optimization used for disaster resilient location detection in mobile ad-hoc network." Journal of Ambient Intelligence and Humanized Computing (2020): 1-11.

[11] Smys, S., and Jennifer S. Raj. "A Stochastic Mobile Data Traffic Model for Vehicular Ad Hoc Networks." Journal of Ubiquitous Computing and Communication Technologies (UCCT) 1, no. 01 (2019): 55-63.

ISSN: 2582-2640 (online)

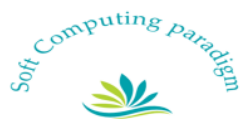


Journal of Soft Computing Paradigm (JSCP) (2020)

Vol.02/ No. 01

Pages: $13-23$

http://irojournals.com/jscp/

DOI: https://doi.org/10.36548/jscp.2020.1.002

[12] Sivaganesan, D. "Efficient Routing Protocol With Collision Avoidance In Vehicular Networks." Journal of Ubiquitous Computing and Communication Technologies (UCCT) 1, no. 02 (2019): 76-86.

[13] Haoxiang, Wang. "Multi-Objective Optimization Algorithm For Power Management In Cognitive Radio Networks." Journal of Ubiquitous Computing and Communication Technologies (UCCT) 1, no. 02 (2019): 97-109.

[14] Smys, S., and Jennifer S. Raj. "Performance Optimization Of Wireless Adhoc Networks With Authentication." Journal of Ubiquitous Computing and Communication Technologies (UCCT) 1, no. 02 (2019): 64-75.

[15] Duraipandian, M. "Performance Evaluation Of Routing Algorithm For Manet Based On The Machine Learning Techniques." Journal of trends in Computer Science and Smart technology (TCSST) 1, no. 01 (2019): 25-38.

[16] Haoxiang, Wang, and S. Smys. "Soft Computing Strategies for Optimized Route Selection in Wireless Sensor Network." Journal of Soft Computing Paradigm (JSCP) 2, no. 01 (2020): 125 137.

[17] Alshamlan, Hala M., Ghada H. Badr, and Yousef A. Alohali. "Genetic Bee Colony (GBC) algorithm: A new gene selection method for microarray cancer classification." Computational biology and chemistry 56 (2015): 49-60.

ISSN: 2582-2640 (online) 